\title{
Wide-Range, Picoampere-Sensitivity Multichannel VLSI Potentiostat for Neurotransmitter Sensing
}

\author{
Kartikeya Murari*, Nitish Thakor* \\ Milutin Stanacevic ${ }^{\dagger}$, and Gert Cauwenberghs ${ }^{\dagger}$ \\ * Department of Biomedical Engineering, Johns Hopkins University School of Medicine, Baltimore, MD 21205 \\ $\dagger$ Department of Electrical and Computer Engineering, Johns Hopkins University, Baltimore, MD 21218 \\ kartik@jhu.edu,nthakor@bme.jhu.edu, miki@jhu.edu, gert@jhu.edu
}

\begin{abstract}
Neurotransmitter sensing is critical in studying nervous pathways and neurological disorders. A 16-channel currentmeasuring VLSI potentiostat with multiple ranges from picoamperes to microamperes is presented for electrochemical detection of electroactive neurotransmitters like dopamine, nitric oxide etc. The analog-to-digital converter design employs a current-mode, first-order single-bit delta-sigma modulator architecture with a two-stage, digitally reconfigurable oversampling ratio for ranging the conversion scale. An integrated prototype is fabricated in CMOS technology, and experimentally characterized. Real-time multi-channel acquisition of dopamine concentration in vitro is performed with a microfabricated sensor array.
\end{abstract}

\section{INTRODUCTION}

Fast, accurate and distributed neurotransmitter measurements are crucial in unravelling how information is carried in the nervous system and understanding mechanisms of several neurological disorders like Parkinson's disease and epilepsy. Numerous methods like optical, immunochemical, liquid chromatography are used to detect and measure neurotransmitter activity. Electrochemical sensing of certain electroactive neurotransmitters (eg nitric oxide, dopamine) is very attractive due to high sensitivity, rapidity and the ability to perform distributed measurements [1], [2], [3].

Electrochemical measurements are based on oxidizing or reducing the neurotransmitters at electrodes held at a potential characteristic for that specific neurotransmitter (the redox potential) with respect to a reference electrode. The neurotransmitter molecules get adsorbed at the electrode surface. This leads to the exchange of one or more electrons between the electrode and the neurotransmitter molecules. These electrons constitute the redox current. The redox currents are directly proportional to the concentration of the species in solution. The currents range from picoamperes to microamperes and have a slow time scale ranging from milliseconds to seconds.

Currently these measurements are done using a potentiostat (Model 273A, Princeton Applied Research, Oak Ridge, TN) a large bench-top instrument that is capable of measuring current while maintaining a constant (redox) potential. The bulk of the instrument makes multichannel recordings cumbersome and expensive. The ability to record neurotransmitter levels from a population of neurons, rather than a single cell, is of tremendous potential. Sensor arrays can be microfabricated [4], [5], [6] to perform high spatial resolution sensing. Accordingly compact multichannel potentiostats are needed

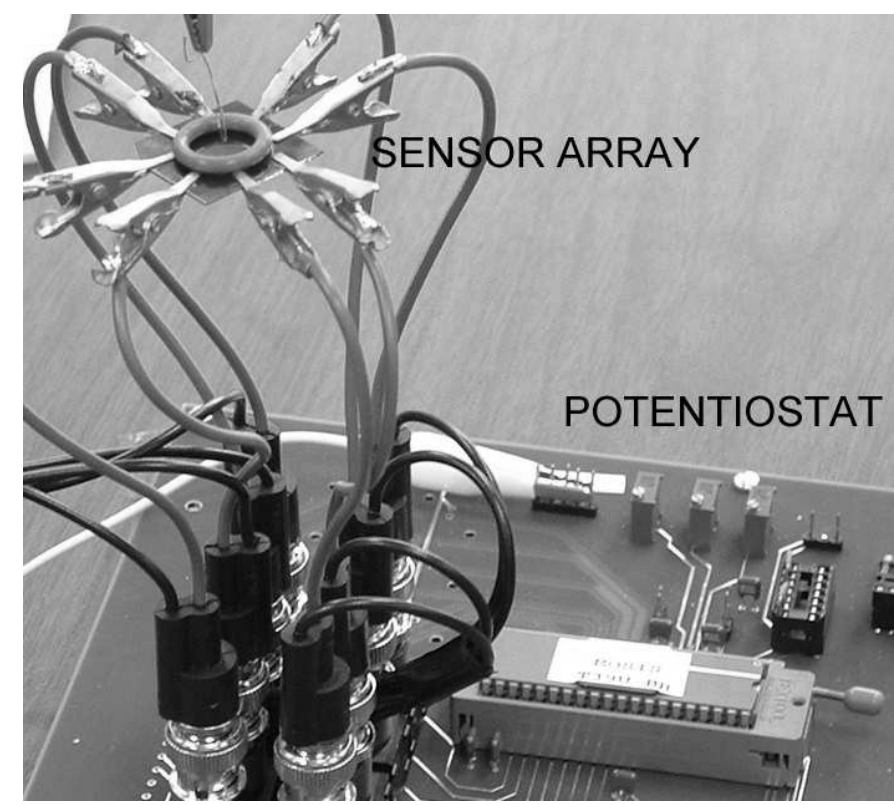

Fig. 1. Experimental setup showing the potentiostat chip acquiring 8 channel real-time neurotransmitter measurements with the sensor array.

to be able to process the signals from the sensor arrays. VLSI technology is particularly attractive here for low-power,

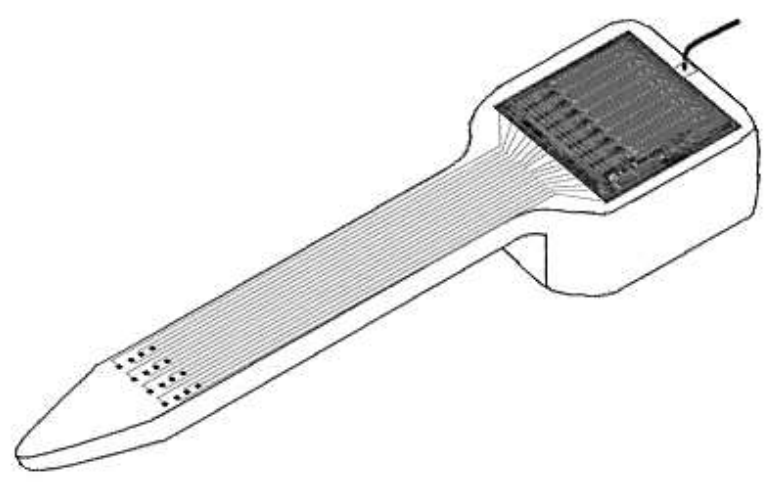

Fig. 2. Envisioned implantable probe with integrated VLSI potentiostat (on the base of the probe) and electrochemical sensors (on the shank of the probe). 


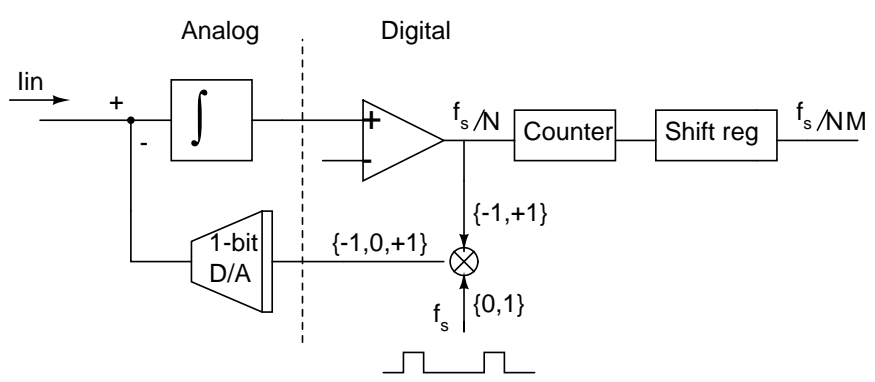

Fig. 3. Block diagram of 1 channel of potentiostat array.

multichannel systems. Integrated potentiostats with one or few parallel channels have been previously reported by others [7], [8] and our group [9], [10].

Our current system is shown in Figure 1. Integrated VLSI multichannel potentiostats of the kind presented can be integrated along with the sensor arrays developed by our group [6] to fabricate a implantable neurotransmitter monitoring device shown in Figure 2. Such a device will have an extremely powerful impact on the current state of understanding neural pathways and neurological diseases.

\section{Chip ARChitecture}

The currents of interest range from picoamperes to microamperes, with time scales ranging from miliseconds to seconds. The current span of several orders of magnitude calls for different scales of measurements and the long time constant enables long integration times.

A block diagram of one channel of potentiostat array is shown in Figure 3. The AD converter implemented is current first-order single bit delta-sigma modulator. The choice of the modulator is driven by low-frequency content of the signal of interest, which allows high oversampling ratios and trade-off between bandwidth and resolution. The high-frequency noise is reduced in digital domain together with shaped quantization noise. Single-bit quantization leads to very robust circuits, less sensitive to matching and imperfection errors. The firstorder modulator is also amenable to simple and compact implementation. The sampled-data switched-capacitor (SC) realization offers low-noise and low-power implementation.

The measured current is integrated on capacitor with high gain amplifier providing a high input conductance virtual ground node at voltage that can be set by external reference. The reference voltage at the input node is jointly set for 4 channels. To achieve wide dynamic range of currents with multiple scales, as alternative to using imprecise current mirrors, we have chosen to insert a time-modulation in the feedback loop, a variable time-period over which the feedback is active. This introduces a digitally programmable gain of input current with respect to reference current of 1-bit DA converter, as a small input current is to be integrated over longer time period, while a reference current is integrated over shorter time interval. The 1-bit results of comparison between integrated voltage and zero-level is multiplied with the feedback-control

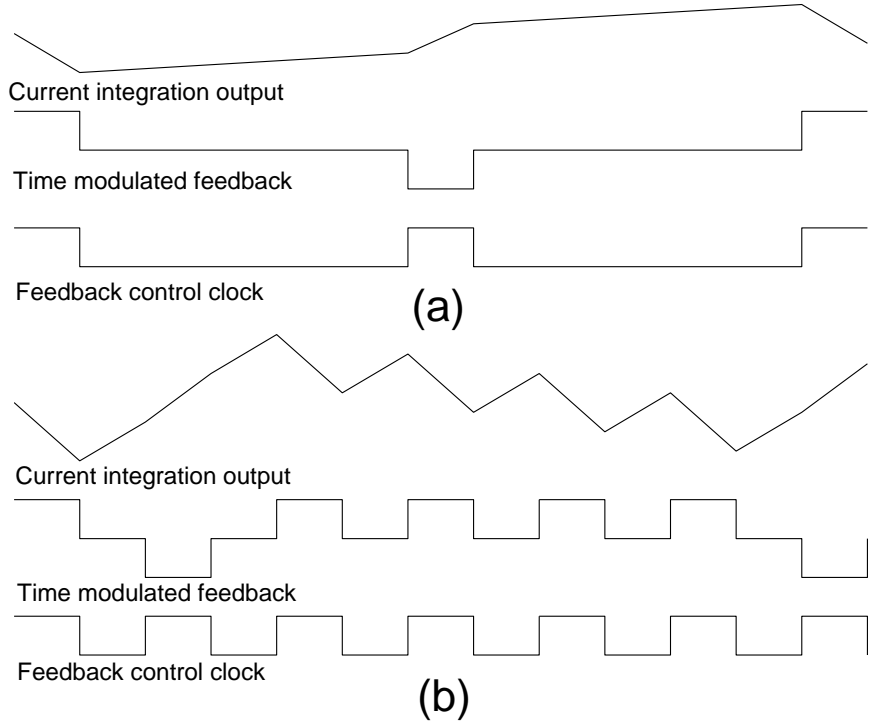

Fig. 4. Time-modulation of the feedback signal for two different values of digital gain $N$.

clock, enabling the feedback loop only when this feedbackcontrol clock is active. The digital gain of the input current $N$ is equal to the duty-cycle of feedback-control clock. This clock is derived from the system clock of frequency $f_{s}$ and the duration of the period when the feedback clock is high is equal to the period of the system clock. The duration of the period when the feedback clock is low is a multiple of period of the system clock. In the Figure 4, we illustrate the feedback-control clock, time-modulated feedback signal and output voltage of the integrator for two different values of digital gain $N$. By adjusting the programmable duty-cycle for the same reference current and system clock frequency, the chip is capable of handling wide range of currents. The gain $N$ can be set anywhere between 1 and 65536 .

The decimator is implemented using binary counter and the digital value is stored in a output register at the end of the conversion cycle. The conversion cycle is variable and can be set anywhere between 1 and 65536 times the period of the feedback-control clock. The output register can be read asynchronously at any time during conversion cycle. The 16 bits representing the digital value of input current of each channel are shifted out bit-serially using clock independent of system clock and 256 cycles are necessary to read out all 16 channels.

\section{CHIP CHARACTERIZATION}

To demonstrate the wide range of input currents that can be measured with the potentiostat chip, we show experimental results for two different gains. The reference current was set to $0.6 \mu \mathrm{A}$ and the system clock frequency was $2 \mathrm{MHz}$. The input potential was set to $1 \mathrm{~V}$. In the first experiment we have set the gain of the input current to 1 which set the input current scale to be from $-0.5 \mu \mathrm{A}$ to $0.5 \mu \mathrm{A}$. The oversampling ratio was set to 65536 leading to conversion time of $33 \mathrm{~ms}$. 


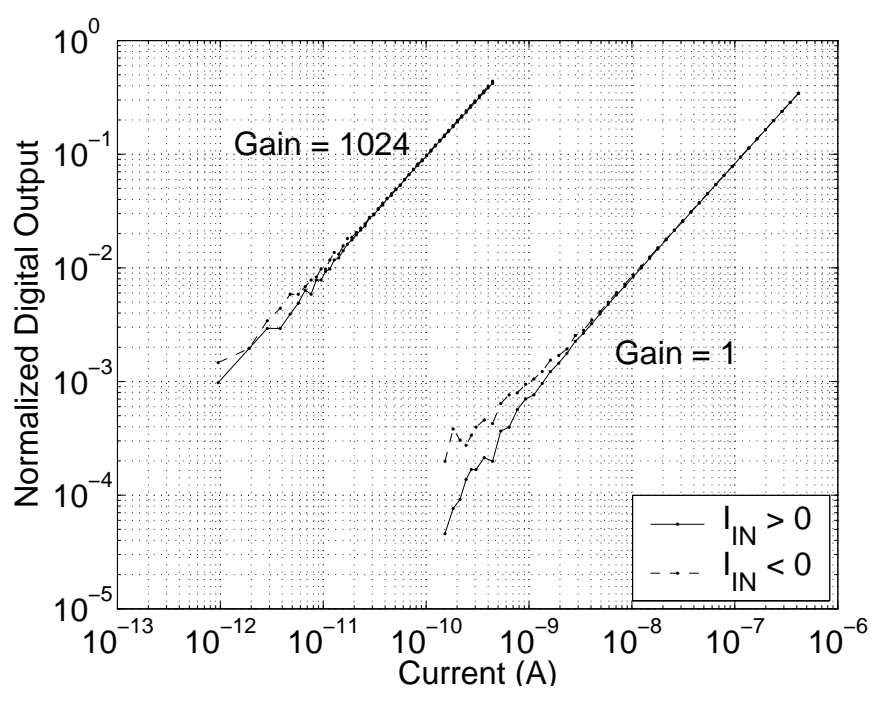

Fig. 5. Normalized digital output as function of the input current for gain of 1 and oversampling ratio of 65536 and gain of 2048 and oversampling ratio of 1024 .

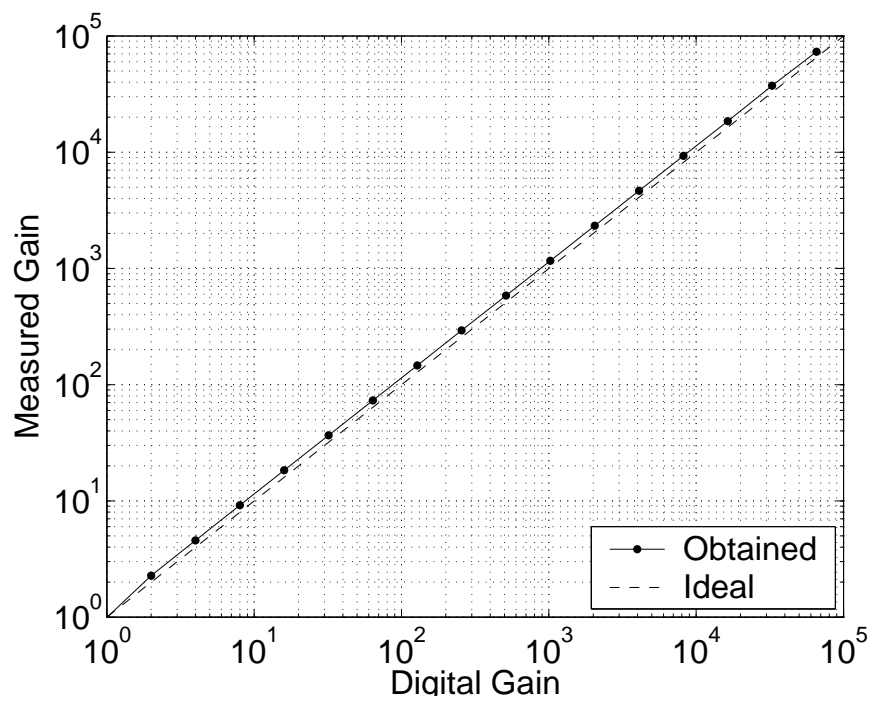

Fig. 6. Experimentally measured gain as function of digitally set gain of input current.

The input current was swept in logarithmically fashion using Keithley SourceMeter model 6430 (Keithley Instruments Inc., Cleveland, $\mathrm{OH})$. In the second experiment the gain of the input current was set to 1024 and the oversampling ratio was set to 2048 leading to conversion time of 1s. Figure 5 shows the normalized digital output of the chip for channel 9 as function of the input current in both cases.

To demonstrate the wide range of digital gains that can be used for setting the different scale, we show in figure 6 experimentally measured gains at different scale defined by digital gain.

\section{Neurotransmitter measurements}

The chip was used for potentiostatic measurements of the neurotransmitter Dopamine in a phosphate buffered solution

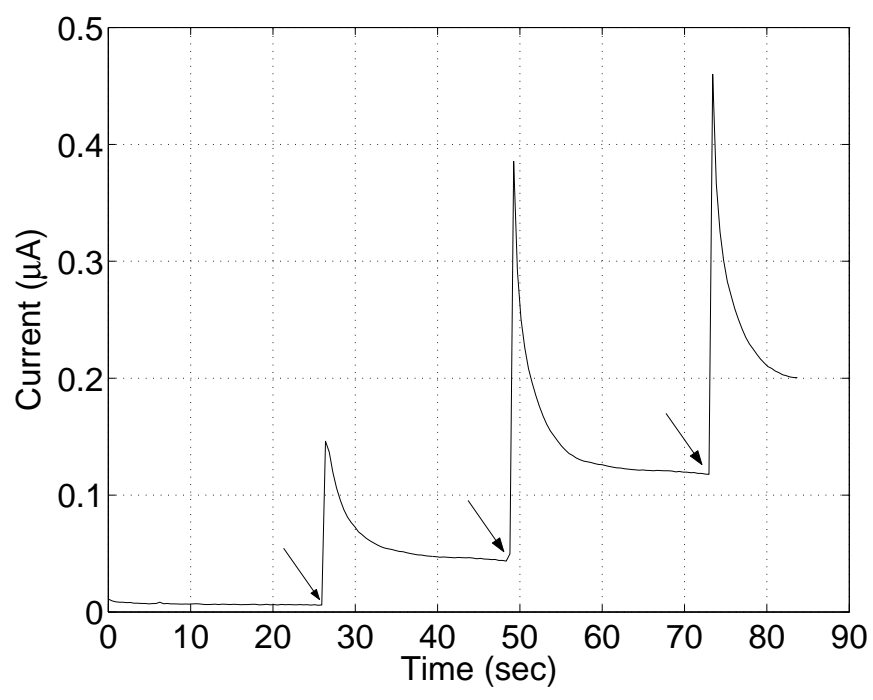

Fig. 7. Real time neurotransmitter monitoring by the chip using the commercial CF $30-250$ as the working electrode. The arrow marks denote times of $1 \mu \mathrm{L}$ dopamine addition.

(PBS). A standardized solution of Dopamine was prepared thus. 20mg of Dopamine Hydrochloride (Alfa Aesar, Ward Hill, MA) was dissolved in $99 \mathrm{~mL}$ of deionized water. $1 \mathrm{~mL}$ of Perchloric Acid (Alfa Aesar, Ward Hill, MA) was added to prevent spontaneous oxidation of Dopamine. The solution thus obtained is $1.055 \mathrm{mM}$. The $\mathrm{Ag} / \mathrm{AgCl}$ reference electrode was made by electrodepositing a layer of $\mathrm{AgCl}$ on a silver wire. This was done by holding a $\mathrm{Ag}$ wire in a solution of $0.1 \mathrm{~N} \mathrm{HCl}$ at a potential of $1 \mathrm{~V}$ with respect to a platinum wire reference electrode for 20 minutes. Two kinds of carbon microsensors were used as working electrodes for testing the chip - a microfabricated $4 \times 4$ array of electrodes fabricated as in [6] and a commercially available carbon microfiber electrode (CF 30-250, WPI Inc, Sarasota, FL). The microfabricated array was used for multi-channel measurements.

The experimental procedure is thus. The working and the reference electrode are immersed in PBS. An oxidation potential of $0.9 \mathrm{~V}$ is set up on the current sensing terminals of the potentiostat chip with respect to the reference electrode and the arrangement is allowed to equilibrate. The serial output of the chip is monitored in real-time and stored on a computer. Boluses of the dopamine solution are added into the PBS at fixed intervals.

Figure 7 shows the chip output for a single channel measurement from the commercial CF 30-250 electrode. Boluses of $1 \mu \mathrm{L}$ of the dopamine solution were added at 25 second intervals to the PBS using a gas-tight syringe. This is shown by the arrow marks in figure 7 . The digital output of the chip was converted into a current using the current versus digital output characteristics of the chip. Figure 8 shows a multi-channel measurement using the microfabricated electrode array [6]. Output from four of the channels is presented for clarity. The boluses were added at 120 second intervals. The nonsymmetric arrangement of the electrodes with respect to the 


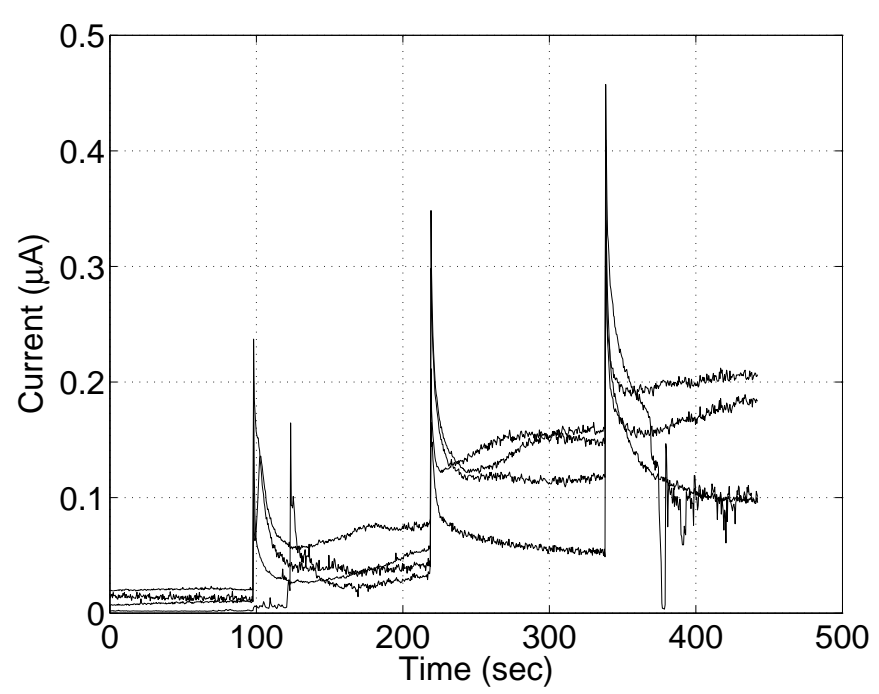

Fig. 8. Real time four channel simultaneous neurotransmitter monitoring by the chip using the microfabricated electrode array as the working electrode.

point of addition of the dopamine boluses and the agitation caused by adding a bolus accounts for the time delays and magnitude variations between the responses from different electrodes.

The difference in the timescales of Figure 7 and Figure 8 is explained on the account of different electrode surfaces of the two kinds of electrodes used. Electrochemical currents depend on the nature of the reactive surface and thus reaction rates vary with electrodes of different kinds.

\section{CONClusion}

We presented a 16-channel potentiostat array with a wide dynamic range of currents that span through six orders of magnitude from picoamperes to microamperes and sensitivity of below $1 \mathrm{pA}$. The current range is controlled through programmable feedback duty-ratio cycle. A commercially available electrochemical sensor was used with the chip as a benchmark for the potentiostat. The potentiostat chip was used to acquire real-time multichannel data from a microfabricated neurotransmitter sensor array as a milestone in our proposed integrated implantable neurotransmitter monitoring system.
Acknowledgments: This work was supported by $\mathrm{NIH}$ MH062444 065296, ONR N00014-99-1-0612, NSF IIS0209289, and the Whitaker Foundation. Chips were fabricated through the MOSIS foundry service.

\section{REFERENCES}

[1] F. Bedioui, S. Trevin, and J. Devynck, "The use of gold electrodes in the electrochemical detection of nitric oxide in aqueous solution", J. Electroanal. Chem., vol. 377, pp. 295, 1994.

[2] T. Malinski, Z. Taha, S. Grunfeld, A. Burewicz, and P. Tomboulian, "Measurements of nitric oxide in biological materials using a porphyrinic microsensor", Anal. Chim. Acta., vol. 279, pp. 135, 1994.

[3] R.B. Kawade and K.S.V. Santhanam, "An in vitro electrochemical sensing of dopamine in the presence of ascorbic acid", Biochemistry and Bioenergetics, vol. 38, pp. 405, 1995.

[4] J.K. Park, P.H. Tran, J.K.T. Chao, R. Godhara, and N.V. Thakor, "In Vivo Nitric Oxide Sensor Using Non-Conducting Polymer Modified Carbon Fiber," Biosensors Bioelectronics, vol. 13, pp. 1187, 1998.

[5] P.M. George, J. Muthuswamy, J. Currie, N.V. Thakor, and m. Paranjape, "Fabrication of screen-printed carbon electrodes for sensing neuronal messengers," BioMEMS, vol. 3 (4), pp. 307, 2001.

[6] M. Naware, N.V. Thakor, R.N. Orth, K. Murari, and P.A. Passeraub, "Design and Microfabrication of a Polymer Modified Carbon Sensor Array for the Measurement of Neurotransmitter Signals," 25th Annual International Conference of the IEEE EMBS, Washington DC, 2003

[7] R.B.F. Turner, D.J. Harrison, and H.P. Baltes, "A CMOS Potentiostat for Amperometric Chemical Sensors," IEEE J. Solid-State Circuits, vol. SC-22, pp. 473-478, 1987.

[8] R.G. Kakerow, H. Kappert, E. Spiegel, and Y. Manoli, "Low Power Single Chip CMOS Potentiostat," Transducers 95, Eurosensors IX, vol. 1, pp. 142-145, 1995.

[9] A. Bandyopadhyay, G. Mulliken, G. Cauwenberghs, and N. Thakor, "VLSI Potentiostat Array for Distributed Electrochemical Neural Recording," Proc. IEEE Int. Symp. Circuits and Systems (ISCAS'2002), Phoenix AZ, May 26-29, 2002.

[10] G. Mulliken, M. Naware, A. Bandyopadhyay, G. Cauwenberghs and N. Thakor, "Distributed Neurochemical Sensing: In Vitro Experiments," Proc. IEEE Int. Symp. Circuits and Systems (ISCAS'2003), Bangkok Thailand, May 25-28, 2003.

[11] J. Candy and G. Temes, "Oversampling delta-sigma data converters", IEEE Press, 1991. 